\title{
WEAK POSITIVE MATRICES AND HYPONORMAL WEIGHTED SHIFTS
}

\author{
H. EL-AZHAR, K. IDRISSI, E.H. ZEROUALI
}

\begin{abstract}
In the paper we study $k$-positive matrices, that is, the class of Hankel matrices, for which the $(k+1) \times(k+1)$-block-matrices are positive semi-definite. This notion is intimately related to a $k$-hyponormal weighted shift and to Stieltjes moment sequences. Using elementary determinant techniques, we prove that for a $k$-positive matrix, a $k \times k$ block-matrix has non zero determinant if and only if all $k \times k$-block matrices have non zero determinant. We provide several applications of our main result. First, we extend the Curto-Stampfly propagation phenomena for for 2-hyponormal weighted shift $W_{\alpha}$ stating that if $\alpha_{k}=\alpha_{k+1}$ for some $n \geqslant 1$, then for all $n \geqslant 1, \alpha_{n}=\alpha_{k}$, to $k$-hyponormal weighted shifts to higher order. Second, we apply this result to characterize a recursively generated weighted shift. Finally, we study the invariance of $k$-hyponormal weighted shifts under one rank perturbation. A special attention is paid to calculating the invariance interval of 2-hyponormal weighted shift; here explicit formulae are provided.
\end{abstract}

Keywords: Subnormal operators, $k$-hyponormal operators, $k$-positive matrices, weighted shifts, perturbation, moment problem.

Mathematics Subject Classification: 47B37, 44A60

\section{INTRODUCTION}

Let $\mathcal{H}$ be a complex Hilbert space and let $\mathcal{L}(\mathcal{H})$ be the algebra of bounded operators on $\mathcal{H}$. We denote by $[T, S]:=T S-S T$ the commutator of $S$ and $T$ in $\mathcal{L}(\mathcal{H})$. An operator $T \in \mathcal{L}(\mathcal{H})$ is said to be normal if $\left[T^{*}, T\right]=0$, to be hyponormal if $\left[T^{*}, T\right] \geqslant 0$ and to be subnormal if $T=N_{\mid \mathcal{H}}$, where $N$ is a normal operator on some Hilbert space $\mathcal{K} \supseteq \mathcal{H}$.

The concepts of subnormal and hyponormal operators were introduced by Paul R. Halmos in [1]. The first notion, hyponormal, reflects the geometric nature of normality with the corresponding implications in terms of positive matrices; while subnormal is intimately related to the notion of analyticity for complex functions through the restriction of the functional calculus to invariant subspaces.

In order to establish a bridge between the operator theory and matrix theory, we recall the BramHalmos criterion for subnormality [2, 3], which says that an operator $T$ is subnormal if and only if

$$
\sum_{i, j \leqslant k}\left\langle T^{i} x_{j}, T^{j} x_{i}\right\rangle \geqslant 0 \quad \text { for all } k \geqslant 0,
$$

for any $x_{0}, x_{1}, \ldots, x_{k} \in \mathcal{H}$. An application of the Choleski algorithm for operator matrices shows that (1.1) is equivalent to the positivity test

H. El-Azhar, K. Idrissi, E.H. Zerouali, Weak positive matrices and hyponormal Weighted SHIFTS.

(c) El-Azhar H., Idrissi K., Zerouali E.H. 2019.

The first and the last author are supported by the Project URAC 03 of the National center of research and by the Hassan II Academy of Sciences and Technologies. The first author is partially supported by the CNRST grant 24UM5R2015, Morocco.

Submitted December 29, 2018. 


$$
M_{k}(T):=\left(\begin{array}{cccc}
{\left[T^{*}, T\right]} & {\left[T^{* 2}, T\right]} & \ldots & {\left[T^{* k}, T\right]} \\
{\left[T^{*}, T^{2}\right]} & {\left[T^{* 2}, T^{2}\right]} & \ldots & {\left[T^{* k}, T^{2}\right]} \\
\vdots & \vdots & \ddots & \vdots \\
{\left[T^{*}, T^{k}\right]} & {\left[T^{* 2}, T^{k}\right]} & \ldots & {\left[T^{* k}, T^{k}\right]}
\end{array}\right) \geqslant 0 \quad \text { for all } k \geqslant 0
$$

To illustrate and to study the gap between subnormal and hyponormal operators, A. Athavale [4] introduced the classes of $k$-hyponormal operators as follows. An operator $T \in \mathcal{L}(\mathcal{H})$ is $k$-hyponormal if $M_{k}(T) \geqslant 0$. Clearly

$$
T \text { is subnormal } \Leftrightarrow T \text { is } k \text {-hyponormal for each } k \in \mathbb{N} \text {. }
$$

Moreover,

$$
(k+1) \text {-hyponormal } \Rightarrow k \text {-hyponormal } \Rightarrow \text {-hyponormal }=\text { hyponormal. }
$$

Weighted shifts defined below provide several examples and counterexamples in the operator theory and hence are an important motivation in the analysis of operators. Given a bounded sequence of positive numbers $\alpha \equiv\left\{\alpha_{n}\right\}_{n \geqslant 0}$ (called weights), the unilateral weighted shift $W_{\alpha}$ associated with $\alpha$ is a bounded operator on $\ell^{2}(\mathbb{N})$ defined by $W_{\alpha} e_{n}:=\alpha_{n} e_{n+1}$ for each $n \geqslant 0$, where $\left\{e_{n}\right\}_{n=0}^{\infty}$ is the canonical orthonormal basis for $\ell^{2}$; the moments of $\alpha$ are defined by $\gamma_{0}:=1, \gamma_{n+1}:=\alpha_{n}^{2} \gamma_{n}(n \geqslant 0)$. It is straightforward to check that $W_{\alpha}$ can never be normal, and that $W_{\alpha}$ is hyponormal if and only if $\alpha_{n} \leqslant \alpha_{n+1}$ for all $n \geqslant 0$.

The Stieltjes moment problem associated with a given sequence $\left\{\gamma_{n}\right\}_{n \geqslant 0}$ entails finding a positive Borel measure $\mu$ supported in $\mathbb{R}_{+}$such that

$$
\gamma_{n}=\int_{\mathbb{R}_{+}} t^{n} d \mu \quad \text { for each } n \geqslant 0 .
$$

When the moment problem possesses a solution $\mu$, then $\mu$ is said to be a representing measure of the moment sequence $\left\{\gamma_{n}\right\}_{n \geqslant 0}$. The well known Berger theorem says that a weighted shift $W_{\alpha}$ is subnormal precisely when the sequence of its moments is a moment sequence of a positive measure supported in $\left[0,\left\|W_{\alpha}\right\|\right]$.

A description of subnormality for an abstract operator $T$ in terms of weighted shifts can be found in [5. Namely, a one-to-one operator $T$ is subnormal if and only if, for each $h \neq 0$ in $\mathcal{H}$, the weighted shift associated with the weight sequence $\left\{\left\|T^{n+1} h\right\| /\left\|T^{n} h\right\|\right\}$ is subnormal.

J. Stampfli in [6] (see also [7]) showed that for subnormal weighted shifts $W_{\alpha}$, a propagation phenomenon occurs which forces the flatness of $W_{\alpha}$ whenever two equal weights are present. That is, if $\alpha_{k}=\alpha_{k+1}$ for some $k \geqslant 0$, then $\alpha_{n}=\alpha_{n+1}$ for every $n \geqslant 1$. Later, in [8], R. Curto proved that the above result remains valid for 2 -hyponormal weighted shifts. Our main goal in this note is to generalize the propagation phenomena in order to study the gap between different classes of $k$-hyponormal weighted shifts. To this aim we introduce the notion of $k$-positive matrices (or sequences), and we study this concept to exhibit some useful results. The approach we use here provides clear direct computations, which makes proof much simpler than [8, 9]. It can also lead to more generalizations on perturbation problems of $k$-hyponormal operators.

This paper is organized as follows. In Section 2 we define the concept of $k$-positive matrices, and we give some of their basic properties. In Section 3 we formulate the main results. In Section 4 we give an elementary proof to the propagation phenomena for $k$-positive matrix, that is, a $k$-positive matrix has a $k \times k$-sub-matrix with zero determinant if and only if all $k \times k$-sub-matrices have zero determinant. We devote Section 5 to translate the propagation phenomena in term of $k$-hyponormal weighted shifts. In the last section we study the invariance of $k$-hyponormal weighted shifts under one rank perturbation, and a simple algorithm for calculating the stable perturbation intervals. 


\section{$2 . \quad k$-POSITIVE HANKEL MATRICES}

Given a sequence of non negative numbers $\gamma=\left\{\gamma_{n}\right\}_{n \geqslant 0}$, the associated Hankel matrix is built as follows

$$
M_{\gamma}:=\left(\gamma_{i+j}\right)_{i, j}=\left(\begin{array}{cccc}
\gamma_{0} & \gamma_{1} & \gamma_{2} & \gamma_{3} \\
\gamma_{1} & \gamma_{2} & \gamma_{3} & \ldots \\
\gamma_{2} & \gamma_{3} & \ldots & \ldots \\
\gamma_{3} & \vdots & \ddots & \ddots
\end{array}\right) .
$$

For $k, n \in \mathbb{N}$, we denote by $\left[M_{\gamma}\right]_{k}^{n}$ the Hankel $(k+1) \times(k+1)$-sub-matrix

$$
\left[M_{\gamma}\right]_{k}^{n}=\left(\begin{array}{cccc}
\gamma_{n} & \gamma_{n+1} & \ldots & \gamma_{n+k} \\
\gamma_{n+1} & \gamma_{n+2} & \ldots & \gamma_{n+k+1} \\
\vdots & \ddots & \ddots & \vdots \\
\gamma_{n+k} & \gamma_{n+k+1} & \ldots & \gamma_{n+2 k}
\end{array}\right) .
$$

Definition 2.1. A Hankel matrix $M_{\gamma}$, or a sequence $\gamma$, is said to be $k$-positive if for every $n \in \mathbb{N}$, the $(k+1) \times(k+1)$-sub-matrix $\left[M_{\gamma}\right]_{k}^{n}$ is positive semi-definite.

Clearly, $M_{\gamma}$ is $k$-positive means that $\left\langle M_{\gamma} x, x\right\rangle \geqslant 0$ for each $x=\sum_{i=0}^{k} x_{i} e_{n+i}$ and $n \in \mathbb{N}$. Also, it is easy to see that the set of $k$-positive matrices, denoted by $\mathcal{C}_{+}^{k}$, is a convex cone, and that $\mathcal{C}_{+}^{k+1} \subset \mathcal{C}_{+}^{k}$ for all $k \in \mathbb{N}$.

Further immediate properties and examples are given in the next remark:

\section{Remark 1.}

1. $\mathcal{C}_{+}^{0}$ is the set of all matrices with non-negative entries.

2. $\gamma=\left\{\gamma_{n}\right\}_{n \geqslant 0}$ is 1-positive if and only if $\gamma$ is non-negative and log-convex.

3. Let $\mu$ be a positive finite measure such that Supp $(\mu) \subset \mathbb{R}_{+}$and $\mathbb{R}[X] \subset L^{1}\left(\mathbb{R}_{+}, \mu\right)$. By Stieltjes's Theorem [10] for every $k \in \mathbb{N}$, the Hankel matrices $\left(\gamma_{i+j}\right)_{0 \leqslant i, j \leqslant k}$ and $\left(\gamma_{i+j+1}\right)_{0 \leqslant i, j \leqslant k}$ are positives semi-definite. In other words, $\gamma$ is $k$-positive for all $k \in \mathbb{N}$.

\section{MAIN RESULTS}

From the log-convexity of $k$-positive sequences, we deduce the next useful statement.

Proposition 1. Let $M \in \mathcal{C}_{+}^{k}$. If $\gamma_{n_{0}}=0$, for some $n_{0} \in \mathbb{N}$, then $\gamma_{n}=0$ for every $n \geqslant 1$.

Proposition 1 states that a propagation phenomena occurs, in the sense that if a term of our sequence is zero, then almost all the sequence is forced to be zero. The general case of higher order propagation was established by R. Curto and L. Fialkow in [11, Proposition 5.13]. Our first contribution in this section is to provide an elementary proof of this fact based on a version of block matrices determinants.

Theorem 3.1 (Propagation phenomena for $k$-positive matrices). Let $M \in \mathcal{C}_{+}^{k}$ be such that there exists an integer $n_{0} \geqslant 0$ satisfying $\operatorname{det}\left(\left[M_{\gamma}\right]_{k-1}^{n_{0}}\right)=0$. Then $\operatorname{det}\left(\left[M_{\gamma}\right]_{k-1}^{n}\right)=0$ for all $n \geqslant 1$.

In terms of weighted shifts, the propagation phenomena is formulated as follows.

Theorem 3.2. Let $\alpha=\left\{\alpha_{n}\right\}_{n \geqslant 0}$ be a sequence of positive numbers such that the weighted shift $W_{\alpha}$ is k-hyponormal. We assume that $\operatorname{det}\left(\left[M_{\gamma}\right]_{p}^{n_{0}}\right)=0$ for some $n_{0} \geqslant 0, p<k$, then,

$$
\operatorname{det}\left(\left[M_{\gamma}\right]_{p}^{n}\right)=0 \quad \text { for all } n \geqslant 1 \text {. }
$$

In particular, $W_{\alpha}$ is subnormal.

Let $W_{\alpha}$ be a weighted shift, and let $\gamma=\left\{\gamma_{n}\right\}_{n \geqslant 0}$ be its moment sequence. We will say that $W_{\alpha}$ is recursively generated if there exist $r \in \mathbb{N}^{*}, a_{0}, \cdots, a_{r-1} \in \mathbb{R}$ such that for every $k \geqslant 0$,

$$
\gamma_{k+r}=a_{r-1} \gamma_{k+r-1}+\cdots+a_{1} \gamma_{k+1}+a_{0} \gamma_{k} .
$$

Using Theorem 3.2 , we obtain the following recursiveness criterion for subnormal weighted shifts. 
Theorem 3.3 (recursively generated subnormal weighted shift). Let $W_{\alpha}$ be a subnormal weighted shift and let $\gamma=\left\{\gamma_{n}\right\}_{n \geqslant 0}$ be its moment sequence. Then the following conditions are equivalent:

(i) $W_{\alpha}$ is recursively generated,

(ii) there exist $n_{0}, k \in \mathbb{N}$ such that $\operatorname{det}\left(\left[M_{\gamma}\right]_{k}^{n_{0}}\right)=0$.

Let $W_{\alpha}$ be a $k$-hyponormal weighted shift. A rank one perturbation of $W_{\alpha}$ associated with $i \in \mathbb{N}$ and $t>0$ is the weighted shift $W_{\alpha(l, t)}$ given by, $\alpha(l, t)_{i}=\alpha_{i}$ if $i \neq l$ and $\alpha(l, t)_{l}=t \alpha_{l}$. We also associate with $W_{\alpha}$, the $k$-hyponormal stable set defined by

$$
I^{k}=\left\{t \geqslant 0 ; W_{\alpha(l, t)} \text { is } k \text {-hyponormal }\right\} .
$$

Clearly $1 \in I^{k}$. Furthermore,

Theorem 3.4 (Rank-one perturbation). $I^{k}$ is a nonempty bounded closed interval.

The last result concerns local perturbation, that is, as there exist $\epsilon>0$ such that $] 1-\epsilon, 1+\epsilon\left[\subset I^{k}\right.$. More precisely, we get:

Theorem 3.5 (local perturbation). Let $W_{\alpha}$ be a k-hyponormal weighted shift. Then,

$$
1 \in \stackrel{\circ}{\widehat{I}^{k}} \quad \Longleftrightarrow \quad\left[M_{\gamma}\right]_{k}^{n} \text { is positive definite } \forall n \leqslant k .
$$

\section{Proof of Theorem 3.1}

We start by the introduction of some notations before expanding the proof of Theorem 3.1 .

For an $(n+1) \times(n+1)$-matrix $M=\left(a_{i, j}\right)_{0 \leqslant i, j \leqslant n}$, and for $i_{0}, j_{0} \leqslant n$, we denote by $M\left(\begin{array}{c}i_{0} \\ j_{0}\end{array}\right)$ the $n \times n$-matrix resulting by removing the $i_{0}+1$ row and $j_{0}+1$ column. We also use the notation $M\left(\begin{array}{ll}0 & n \\ 0 & n\end{array}\right)$ for the $(n-1) \times(n-1)$-matrix resulting by removing the first and the last row and column. In the case where $j_{0}=0\left(\operatorname{resp} j_{0}=n\right)$, we simply denote $M\left(\begin{array}{c}i_{0} \\ 0\end{array}\right)$ by $M\left(\widetilde{i_{0}}\right)\left(\operatorname{resp} M\left(\begin{array}{c}i_{0} \\ n\end{array}\right)\right.$ by $\left.M\left(\hat{i_{0}}\right)\right)$.

The next lemma is the key of our proof. It provides the next interesting expansion formula,

Lemma 4.1 (Desnanot-Jacobi adjoint matrix). If $M=\left(a_{i, j}\right)_{0 \leqslant i, j \leqslant n}$ is an $(n+1) \times(n+1)$ matrix, then

$$
\operatorname{det}(M) \operatorname{det}\left(M\left(\begin{array}{ll}
0 & n \\
0 & n
\end{array}\right)\right)=\operatorname{det}(M(\widetilde{0})) \operatorname{det}(M(\widehat{n}))-\operatorname{det}(M(\widehat{0})) \operatorname{det}(M(\widetilde{n})) .
$$

For the proof of this lemma, we refer to [12].

Proof of Theorem 3.1. Suppose that $\left.\operatorname{det}\left(\left[M_{\gamma}\right]_{k-1}^{i}\right]\right)=0$, for some $i \geqslant n_{0}$, and let us show that $\left.\operatorname{det}\left(\left[M_{\gamma}\right]_{k-1}^{j}\right]\right)=0$ for every $j \in \mathbb{N} \backslash\{0\}$. We shall prove that

$$
\left.\left.\operatorname{det}\left(\left[M_{\gamma}\right]_{k-1}^{i-1}\right]\right)=\operatorname{det}\left(\left[M_{\gamma}\right]_{k-1}^{i+1}\right]\right)=0 .
$$

For $M=\left[M_{\gamma}\right]_{k}^{i}$, we get

$$
M\left(\begin{array}{ll}
0 & k \\
0 & k
\end{array}\right)=\left[M_{\gamma}\right]_{k-2}^{i+2}, \quad M(\widetilde{0})=\left[M_{\gamma}\right]_{k-1}^{i+2}, \quad M(\widehat{k})=\left[M_{\gamma}\right]_{k-1}^{i}, \quad M(\widehat{0})=M(\widetilde{k})=\left[M_{\gamma}\right]_{k-1}^{i+1} .
$$

Applying the identity (4.1), we obtain

$$
\operatorname{det}\left(\left[M_{\gamma}\right]_{k}^{i}\right) \operatorname{det}\left(\left[M_{\gamma}\right]_{k-2}^{i+2}\right)=\operatorname{det}\left(\left[M_{\gamma}\right]_{k-1}^{i}\right) \operatorname{det}\left(\left[M_{\gamma}\right]_{k-1}^{i+2}\right)-\operatorname{det}\left(\left[M_{\gamma}\right]_{k-1}^{i+1}\right)^{2} .
$$

Hence, $\operatorname{det}\left(\left[M_{\gamma}\right]_{k-2}^{i+2}\right) \operatorname{det}\left(\left[M_{\gamma}\right]_{k}^{i}\right) \geqslant 0$, and therefore

$$
0=\operatorname{det}\left(\left[M_{\gamma}\right]_{k-1}^{i}\right) \operatorname{det}\left(\left[M_{\gamma}\right]_{k-1}^{i+2}\right) \geqslant \operatorname{det}\left(\left[M_{\gamma}\right]_{k-1}^{i+1}\right)^{2} .
$$

Now $M_{\gamma} \in \mathcal{C}_{+}^{k}$ implies that $\operatorname{det}\left(\left[M_{\gamma}\right]_{k-1}^{i+1}\right)=0$. Finally, $\operatorname{det}\left(\left[M_{\gamma}\right]_{k-1}^{n}\right)=0$ for each $n \geqslant n_{0}$.

Replacing $i$ by $i-2$ in Equation (4.3), we obtain

$$
0=\operatorname{det}\left(\left[M_{\gamma}\right]_{k-1}^{i-2}\right) \operatorname{det}\left(\left[M_{\gamma}\right]_{k-1}^{i}\right) \geqslant \operatorname{det}\left(\left[M_{\gamma}\right]_{k-1}^{i-1}\right)^{2} .
$$

This proves that $\operatorname{det}\left(\left[M_{\gamma}\right]_{k-1}^{i-1}\right)=0$ and therefore $\operatorname{det}\left(\left[M_{\gamma}\right]_{k-1}^{j}\right)=0$ for each $j \geqslant 1$. This completes the proof. 


\section{PROPAGATION PHENOMENA FOR $k$-HYPONORMAL WEIGHTED SHIFTS}

We recall the Stampfli propagation result for subnormal weighted shifts [6, Theorem 6] (see also [7, Proposition 4.5]):

Theorem 5.1. Let $W_{\alpha}$ be a injective hyponormal weighted shift and assume that $W_{\alpha}$ is subnormal and that $\alpha_{i_{0}}=\alpha_{i_{0}+1}$ for some arbitrary $\alpha_{0}$. Then

$$
\alpha_{i}=\alpha_{i+1} \quad \text { for all } i \geqslant 1 .
$$

In terms of moment sequence associated to $W_{\alpha}$, we can reformulate the result as follows.

Theorem 5.2. Let $W_{\alpha}$ be a subnormal weighted shift, and let $\gamma$ be the associated moment sequence. Under the assumption $\left|\begin{array}{cc}\gamma_{i_{0}} & \gamma_{i_{0}+1} \\ \gamma_{i_{0}+1} & \gamma_{i_{0}+2}\end{array}\right|=0$ for some $i_{0} \geqslant 0$, we have

$$
\left|\begin{array}{cc}
\gamma_{i} & \gamma_{i+1} \\
\gamma_{i+1} & \gamma_{i+2}
\end{array}\right|=0, \text { for all } i \geqslant 1
$$

The first extension of the previous propagation notion relaxes subnormality and was given by Curto in $[8$. More precisely:

Theorem 5.3 ([8, Corollary 6]). Let $W_{\alpha}$ be a 2-hyponormal weighted shift and assume that $\alpha_{i_{0}}=\alpha_{i_{0}+1}$. Then

$$
\alpha_{i}=\alpha_{i+1} \text { for all } i \geqslant 1 \text {, and } \alpha_{0} \text { is arbitrary. }
$$

In view of this results, we shall extend the notion of propagation phenomena for $k$-hyponormal weighted shifts. Recall that if $W_{\alpha}$ is a weighted shift with bounded weight sequence $\alpha=\left\{\alpha_{n}\right\}_{n \geqslant 0}$, the moments of $W_{\alpha}$ are usually defined by $\gamma_{0}:=1$ and $\gamma_{n+1}:=\alpha_{n}^{2} \gamma_{n}(n \geqslant 0)$. It is known that (see [8, Theorem 4]) $W_{\alpha}$ is $k$-hyponormal if and only if $\left[M_{\gamma}\right]_{k}^{n} \geqslant 0$ for all $n \geqslant 0$.

Following [11] R. E. Curto and L. Fialkow, a $k$-hyponormal weighted shift is $k$-extremal if it satisfies $\operatorname{det}\left(\left[M_{\gamma}\right]_{k}^{n}\right)=0$ for every $n \geqslant 0$. They proved that such weighted shift are recursively generated [11, Theorem 5.12], by using Šmulian theorem on extension of positive semi-definite matrix. Using the propagation phenomena for $k$-positive matrix we can easily improve the next result due to Curto and Fialkow.

Theorem 5.4 ([11, Proposition 5.13]). Let $\alpha=\left\{\alpha_{n}\right\}_{n \geqslant 0}$ be a sequence of positive numbers such that the weighted shift $W_{\alpha}$ is k-hyponormal. Assume that $\operatorname{det}\left(\left[M_{\gamma}\right]_{p}^{n_{0}}\right)=0$ for some $n_{0} \geqslant 0$ and $k<p$, then

In particular, $W_{\alpha}$ is subnormal.

$$
\operatorname{det}\left(\left[M_{\gamma}\right]_{p}^{n}\right)=0 \quad \text { for all } \quad n \geqslant 1
$$

Proof. Since $W_{\alpha}$ is $k$-hyponormal if and only if $M_{\gamma}$ is $k$-positive, it is $(p+1)$-positive. Since we also have $\operatorname{det}\left(\left[M_{\gamma}\right]_{p}^{n_{0}}\right)=0$, Theorem 3.1 implies that $\operatorname{det}\left(\left[M_{\gamma}\right]_{p}^{n}\right)=0$ for all $n \geqslant 1$. Therefore, $\operatorname{det}\left(\left[M_{\gamma}\right]_{l}^{n}\right)=0$ for all $l \geqslant k$ and $n \geqslant 0$. It follows that $\left[M_{\gamma}\right]_{l}^{n}$ is positive semi-definite for all $l \geqslant k$ and $n \geqslant 0$, and in particular, that $W_{\alpha}$ is subnormal.

For $k=2$, the next extension of Stampfli's propagation result [7, Proposition 4.5] can be found in [8].

Corollary 1. [8, Corollary 6] Let $\alpha=\left\{\alpha_{n}\right\}_{n \geqslant 0}$ be a bounded sequence of positive numbers associated with a 2-hyponormal weighted shift. If $\alpha_{n_{0}}=\alpha_{n_{0}+1}$ for some $n_{0} \geqslant 0$, then $\alpha_{n}=\alpha_{n_{0}}$ for all $n \geqslant 1$. In particular, $W_{\alpha}$ is subnormal.

We recall that a measure $\mu$ is said to be of finite mass point if $\mu$ has finite support or equivalently, $\mu$ is a finite combination of Dirac measures.

The question of characterizing finite mass measure in terms of moments has been intensively studied and arises in several branches, where finite interpolation is needed. It is closely related to a truncated moment problem. For charges (non necessary positive measures), we can see Corollary 4.3 in [14. In the case of positive measures on $\mathbb{R}$, a more recent work of C. Berg and D. Szwarc provides an interesting study, see Theorem 1.1 of [13]. We prove Theorem 3.3 that gives a simple characterization of finite mass measure on $\mathbb{R}^{+}$. 
Proof of Theorem 3.3. $(i) \Rightarrow$ (ii) If we suppose that $W_{\alpha}$ is recursively generated, the there exists $k \geqslant 0$ and $a_{0}, a_{1}, \ldots, a_{k} \in \mathbb{R}$ such that

$$
\gamma_{p+k+1}=\sum_{j=0}^{k} a_{j} \gamma_{p+j} \quad \text { for each } \quad p \in \mathbb{N} .
$$

This yields that $\operatorname{det}\left(\left[M_{\gamma}\right]_{k+1}^{p}\right)=0$.

$(i i) \Rightarrow(i)$ Since $W_{\alpha}$ is subnormal, we conclude that in particular, $W_{\alpha}$ is $k+1$-hyponormal. Then by the condition $\operatorname{det}\left(\left[M_{\gamma}\right]_{k}^{n_{0}}\right)=0$ and Theorem 5.4, we obtain

$$
\operatorname{det}\left(\left[M_{\gamma}\right]_{k+1}^{n}\right)=0 \quad \text { for each } n \in \mathbb{N} \text {. }
$$

Hence, there exists $a_{0}, a_{1}, \ldots, a_{k} \in \mathbb{R}$ such that

$$
\gamma_{p+k+1}=\sum_{j=0}^{k} a_{j} \gamma_{p+j} \quad \text { for each } \quad p \in \mathbb{N} .
$$

Finally, $W_{\alpha}$ is recursively generated. The proof is complete.

\section{Perturbation of K-Positive matrices}

Let $M \in \mathcal{C}_{+}^{k}(H)\left(M=M_{\gamma}\right)$. For $l \in \mathbb{N}^{*}$ and $t \geqslant 0$ we denote by $M_{\gamma^{\prime}}$ the perturbed Hankel matrix whose entries are given by

where

$$
\gamma_{n}^{\prime}=\left\{\begin{array}{lll}
\gamma_{n} & \text { if } & n \leqslant l \\
t \gamma_{n} & \text { if } & n \geqslant l+1
\end{array}\right.
$$

$$
M_{\gamma^{\prime}}=\left(\begin{array}{ccccccc}
\gamma_{0} & \gamma_{1} & \ldots & \gamma_{l} & t \gamma_{l+1} & t \gamma_{l+2} & \ldots \\
\gamma_{1} & \ldots & \gamma_{l} & t \gamma_{l+1} & t \gamma_{l+2} & \ldots & \ldots \\
\vdots & \ldots & t \gamma_{l+1} & t \gamma_{l+2} & \ldots & \ldots & \ldots \\
\gamma_{l} & t \gamma_{l+1} & t \gamma_{l+2} & \ldots & \ldots & \ldots & \ldots \\
t \gamma_{l+1} & t \gamma_{l+2} & \ldots & \ldots & \ldots & \ldots & \ldots \\
t \gamma_{l+2} & \ldots & \ldots & \ldots & \ldots & \ldots & \ldots \\
\vdots & \ldots & \ldots & \ldots & \ldots & \ldots & \ldots
\end{array}\right)
$$

The main goal of this section is to determine when such perturbation of $k$-positive matrix remains $k$-positive.

We notice that for $n \geqslant l+1$, we get $\left[M_{\gamma^{\prime}}\right]_{k}^{n}=t\left[M_{\gamma}\right]_{k}^{n}$ and we deduce that

$$
M_{\gamma^{\prime}} \text { is k-positive } \Longleftrightarrow\left[M_{\gamma^{\prime}}\right]_{k}^{n} \text { is positive semi-definite } \forall n \leqslant l \text {. }
$$

For $n \leqslant l$, we write

with

$$
\left[M_{\gamma^{\prime}}\right]_{k}^{n}=t\left[M_{\gamma}\right]_{k}^{n}+(1-t) H_{k}^{n}(l)
$$

$$
H_{k}^{n}(l)=\left(\begin{array}{ccccccc}
\gamma_{n} & \gamma_{n+1} & \cdots & \gamma_{l} & 0 & \cdots & 0 \\
\gamma_{n+1} & \gamma_{n+2} & \cdots & 0 & 0 & \cdots & 0 \\
\vdots & \vdots & \ddots & \vdots & & \cdots & 0 \\
\gamma_{l} & 0 & \ddots & \vdots & & \cdots & 0 \\
0 & \vdots & \ddots & \vdots & & \cdots & 0 \\
\vdots & \vdots & \ddots & \vdots & & \cdots & 0 \\
0 & 0 & 0 & 0 & 0 & 0 & 0
\end{array}\right)
$$

and we denote

$$
I_{n}^{k}=\left\{t \geqslant 0: \quad t\left[M_{\gamma}\right]_{k}^{n}+(1-t) H_{k}^{n}(l) \text { is positive semi-definite }\right\} .
$$

We have the following property.

Proposition 2. For each $n \leqslant l, I_{n}^{k}$ is a non empty closed interval of $\mathbb{R}_{+}$. 
Proof. For $k \in \mathbb{N}$, let $P_{k}^{+}$be the closed convex cone of $(k+1) \times(k+1)$ positive semi definite matrices, and denote

$$
D=\left\{t\left[M_{\gamma}\right]_{k}^{n}+(1-t) H_{k}^{n}(l): \quad t \geqslant 0\right\} .
$$

It is obvious that $D$ is a closed convex subset of $\mathcal{M}_{k+1}$ and then $D \cap P_{k}^{+}$is a closed convex set. Moreover, the mapping $\gamma_{n}^{k}$ given by

$$
\begin{aligned}
\gamma_{n}^{k}: \mathbb{R}_{+} & \longrightarrow D \\
t & \longmapsto t\left[M_{\gamma}\right]_{k}^{n}+(1-t) H_{k}^{n}(l),
\end{aligned}
$$

is a continuous affine function, and hence $I_{k}^{n}=\left(\gamma_{n}^{k}\right)^{-1}\left(D \cap P_{k}^{+}\right)$is a closed interval of $\mathbb{R}_{+}$. Also, $M$ is $k$-positive implies that $\left[M_{\gamma}\right]_{k}^{n}$ is positive for all $n \in \mathbb{N}$, and hence that $1 \in I_{n}^{k}$ for all $n \in \mathbb{N}$. The proof is complete.

From 6.1), a perturbation of $k$-positive matrix remains $k$-positive if and only if $t \in I^{k}:=\cap_{n \leqslant l} I_{n}^{k}$, our second result is the next proposition.

Proposition 3. For each $k \geqslant 1, I^{k}$ is a compact interval.

Proof. By Proposition 2 we have that $I^{k}$ is a closed non empty interval. To show that $I^{k}$ is bounded, we observe that if $\left[M_{\gamma^{\prime}}\right]_{k}^{n}$ is positive semi-definite, then $\left[M_{\gamma^{\prime}}\right]_{k-1}^{n}$ is positive semi-definite. We deduce that $I_{n}^{k} \subset I_{n}^{k-1}$, and by induction we see that $I_{n}^{k} \subset I_{n}^{1}$. Finally, $I^{k} \subset I^{1}$. Hence, $t \in I^{1}$ if and only if $M_{\gamma^{\prime} l-1}^{1}$ and $M_{\gamma^{\prime}}{ }_{l}^{1}$ have non-negative determinants, that is,

$$
\frac{\gamma_{l}^{2}}{\gamma_{l-1} \gamma_{l+1}} \leqslant t \leqslant \frac{\gamma_{l} \gamma_{l+2}}{\gamma_{l+1}^{2}}
$$

Thus, for each $k \geqslant 1, I^{k} \subset I^{1}=\left[\frac{\gamma_{l}^{2}}{\gamma_{l-1} \gamma_{l+1}} ; \frac{\gamma_{l} \gamma_{l+2}}{\gamma_{l+1}^{2}}\right]$ is bounded. The proof is complete.

6.1. Determination of $I^{2}$. The problem of determining $I^{k}$ for $k \geqslant 2$ seems to be complicated. From the previous proof, we see that $I^{1}=\left[\frac{\gamma_{l}^{2}}{\gamma_{l-1} \gamma_{l+1}} ; \frac{\gamma_{l} \gamma_{l+2}}{\gamma_{l+1}^{2}}\right]$. We devote this section to calculating $I^{2}$.

Since $t \in I^{2}$ if and only if the next four matrices $\left[M_{\gamma^{\prime}}\right]_{l-3}^{2},\left[M_{\gamma^{\prime}}\right]_{l-2}^{2},\left[M_{\gamma^{\prime}}\right]_{l-1}^{2}$ and $\left[M_{\gamma^{\prime}}\right]_{l}^{2}$ are positives semi-definite. We exhibit the corresponding conditions in each case :

- $\left[M_{\gamma^{\prime}}\right]_{l-3}^{2} \geqslant 0$ :

$$
\left[M_{\gamma^{\prime}}\right]_{l-3}^{2}=\left(\begin{array}{ccc}
\gamma_{l-3} & \gamma_{l-2} & \gamma_{l-1} \\
\gamma_{l-2} & \gamma_{l-1} & \gamma_{l} \\
\gamma_{l-1} & \gamma_{l} & t \gamma_{l+1}
\end{array}\right)
$$

is positive semi-definite if and only if

$$
\left|\begin{array}{cc}
\gamma_{l-1} & \gamma_{l} \\
\gamma_{l} & t \gamma_{l+1}
\end{array}\right| \geqslant 0,\left|\begin{array}{cc}
\gamma_{l-3} & \gamma_{l-1} \\
\gamma_{l-1} & t \gamma_{l+1}
\end{array}\right| \geqslant 0 \text { and }\left|\begin{array}{ccc}
\gamma_{l-3} & \gamma_{l-2} & \gamma_{l-1} \\
\gamma_{l-2} & \gamma_{l-1} & \gamma_{l} \\
\gamma_{l-1} & \gamma_{l} & t \gamma_{l+1}
\end{array}\right| \geqslant 0 .
$$

This is equivalent to

$$
\left\{\begin{array}{l}
t \geqslant \max \left(\frac{\gamma_{l}^{2}}{\gamma_{l-1} \gamma_{l+1}}, \frac{\gamma_{l-1}^{2}}{\gamma_{l-3} \gamma_{l+1}}\right), \\
t \geqslant \frac{\left|\begin{array}{ccc}
\gamma_{l-3} & \gamma_{l-2} & \gamma_{l-1} \\
\gamma_{l-2} & \gamma_{l-1} & \gamma_{l} \\
\gamma_{l-1} & \gamma_{l} & 0
\end{array}\right|}{\gamma_{l+1}\left|\begin{array}{cc}
\gamma_{l-3} & \gamma_{l-2} \\
\gamma_{l-2} & \gamma_{l-1}
\end{array}\right|}
\end{array}\right.
$$


The last condition is redundant. Indeed, using Lemma 4.1 (also called Dodgson condensation method), we obtain:

$$
\left|\begin{array}{ccc}
\gamma_{l-3} & \gamma_{l-2} & \gamma_{l-1} \\
\gamma_{l-2} & \gamma_{l-1} & \gamma_{l} \\
\gamma_{l-1} & \gamma_{l} & 0
\end{array}\right|=\frac{-1}{\gamma_{l-1}}\left[\gamma_{l}^{2}\left|\begin{array}{cc}
\gamma_{l-3} & \gamma_{l-2} \\
\gamma_{l-2} & \gamma_{l-1}
\end{array}\right|+\left|\begin{array}{cc}
\gamma_{l-2} & \gamma_{l-1} \\
\gamma_{l-1} & \gamma_{l}
\end{array}\right|^{2}\right] \leqslant 0 .
$$

By using a simple observation

$$
\frac{\gamma_{l}^{2}}{\gamma_{l-1} \gamma_{l+1}}=\frac{\gamma_{l-1}^{2}}{\gamma_{l-3} \gamma_{l+1}} \frac{\gamma_{l-3} \gamma_{l-1}}{\gamma_{l-2}^{2}}\left(\frac{\gamma_{l-2} \gamma_{l}}{\gamma_{l-1}^{2}}\right)^{2} \geqslant \frac{\gamma_{l-1}^{2}}{\gamma_{l-3} \gamma_{l+1}}
$$

we get that

$$
\left[M_{\gamma^{\prime}}\right]_{l-3}^{2} \text { is positive semidefinite if and only if } t \geqslant \frac{\gamma_{l}^{2}}{\gamma_{l-1} \gamma_{l+1}} .
$$

- $\left[M_{\gamma^{\prime}}\right]_{l-2}^{2} \geqslant 0$ : Using the same argument for

$$
\left[M_{\gamma^{\prime}}\right]_{l-2}^{2}=\left(\begin{array}{ccc}
\gamma_{l-2} & \gamma_{l-1} & \gamma_{l} \\
\gamma_{l-1} & \gamma_{l} & t \gamma_{l+1} \\
\gamma_{l} & t \gamma_{l+1} & t \gamma_{l+2}
\end{array}\right)
$$

we obtain:

$$
\left|\begin{array}{cc}
\gamma_{l-2} & \gamma_{l} \\
\gamma_{l} & t \gamma_{l+2}
\end{array}\right| \geqslant 0, \quad\left|\begin{array}{cc}
\gamma_{l} & \gamma_{l+1} \\
t \gamma_{l+1} & \gamma_{l+2}
\end{array}\right| \geqslant 0, \quad\left|\begin{array}{ccc}
\gamma_{l-2} & \gamma_{l-1} & \gamma_{l} \\
\gamma_{l-1} & \gamma_{l} & t \gamma_{l+1} \\
\gamma_{l} & t \gamma_{l+1} & t \gamma_{l+2}
\end{array}\right| \geqslant 0 .
$$

This implies:

$$
\frac{\gamma_{l}^{2}}{\gamma_{l-2} \gamma_{l+2}} \leqslant t \leqslant \frac{\gamma_{l} \gamma_{l+2}}{\gamma_{l+1}^{2}}
$$

and that the quadric polynomial

$$
P(t):=-\gamma_{l-2} \gamma_{l+1}^{2} t^{2}+\left(\gamma_{l-2} \gamma_{l} \gamma_{l+2}+2 \gamma_{l-1} \gamma_{l} \gamma_{l+1}-\gamma_{l-1}^{2} \gamma_{l+2}\right) t-\gamma_{l}^{3},
$$

is positive. The first and the second inequalities are satisfied immediately since

$$
P\left(\frac{\gamma_{l}^{2}}{\gamma_{l-2} \gamma_{l+2}}\right)=-\frac{\left(\gamma_{l}^{2} \gamma_{l+1}-\gamma_{l-1} \gamma_{l} \gamma_{l+2}\right)^{2}}{\gamma_{l+2}^{2} \gamma_{l-2}} \leqslant 0,
$$

and

$$
P\left(\frac{\gamma_{l} \gamma_{l+2}}{\gamma_{l+1}^{2}}\right)=-\frac{\gamma_{l}\left(\gamma_{l} \gamma_{l+1}-\gamma_{l-1} \gamma_{l+2}\right)^{2}}{\gamma_{l+1}^{2}} \leqslant 0 .
$$

Using the inequality $\gamma_{l-1}^{2} \leqslant \gamma_{l-2} \gamma_{l}$, we see that the second coefficient of $P$ is positive, and hence by the classical Descartes rule for positive roots of polynomials, $P$ has two distinct positive roots. Then

$$
\left[M_{\gamma^{\prime}}\right]_{l-2}^{2} \text { is positive semidefinite if and only if } t \in[\alpha(P) ; \beta(P)],
$$

where $\alpha(P)$ and $\beta(P)$ are the two positive solutions of $P(t)=0$.

- $\left[M_{\gamma^{\prime}}\right]_{l-1}^{2}$ : This case is treated exactly as the last one and leads us to

$$
\left[M_{\gamma^{\prime}}\right]_{l-1}^{2} \text { is positive semidefinite if and only if } t \in[\alpha(Q) ; \beta(Q)],
$$

where $\alpha(Q)$ and $\beta(Q)$ are the two positive solutions of

$$
Q(t):=-\gamma_{l+1}^{3} t^{2}+\left(\gamma_{l-1} \gamma_{l+1} \gamma_{l+3}+2 \gamma_{l} \gamma_{l+1} \gamma_{l+2}-\gamma_{l-1} \gamma_{l+2}^{2}\right) t-\gamma_{l}^{2} \gamma_{l+3}=0
$$


- $\left[M_{\gamma^{\prime}}\right]_{l}^{2}$ : The computations in this case outlines the first one, indeed,

$$
\left[M_{\gamma^{\prime}}\right]_{l}^{2}=\left(\begin{array}{ccc}
\gamma_{l} & t \gamma_{l+1} & t \gamma_{l+2} \\
t \gamma_{l+1} & t \gamma_{l+2} & t \gamma_{l+3} \\
t \gamma_{l+2} & t \gamma_{l+3} & t \gamma_{l+4}
\end{array}\right)
$$

is positive semi-definite if and only if

$$
\left|\begin{array}{cc}
\gamma_{l} & \gamma_{l+1} \\
t \gamma_{l+1} & \gamma_{l+2}
\end{array}\right| \geqslant 0, \quad\left|\begin{array}{cc}
\gamma_{l} & \gamma_{l+2} \\
t \gamma_{l+2} & \gamma_{l+4}
\end{array}\right| \geqslant 0, \quad\left|\begin{array}{ccc}
\gamma_{l} & \gamma_{l+1} & \gamma_{l+2} \\
t \gamma_{l+1} & \gamma_{l+2} & \gamma_{l+3} \\
t \gamma_{l+2} & \gamma_{l+3} & \gamma_{l+4}
\end{array}\right| \geqslant 0 .
$$

This is is equivalent to

$$
t \leqslant \min \left\{\frac{\gamma_{l} \gamma_{l+2}}{\gamma_{l+1}^{2}}, \frac{\gamma_{l} \gamma_{l+4}}{\gamma_{l+2}^{2}}, \frac{-\gamma_{l}\left|\begin{array}{cc}
\gamma_{l+2} & \gamma_{l+3} \\
\gamma_{l+3} & \gamma_{l+4}
\end{array}\right|}{\left|\begin{array}{ccc}
0 & \gamma_{l+1} & \gamma_{l+2} \\
\gamma_{l+1} & \gamma_{l+2} & \gamma_{l+3} \\
\gamma_{l+2} & \gamma_{l+3} & \gamma_{l+4}
\end{array}\right|}\right\}
$$

But

$$
\frac{\gamma_{l} \gamma_{l+2}}{\gamma_{l+1}^{2}}=\frac{\gamma_{l} \gamma_{l+4}}{\gamma_{l+2}^{2}} \frac{\gamma_{l+3}^{2}}{\gamma_{l+4} \gamma_{l+2}}\left(\frac{\gamma_{l+2}^{2}}{\gamma_{l+3} \gamma_{l+1}}\right)^{2} \leqslant \frac{\gamma_{l} \gamma_{l+4}}{\gamma_{l+2}^{2}}
$$

with

$$
\frac{\gamma_{l} \gamma_{l+2}}{\gamma_{l+1}^{2}}+\frac{\gamma_{l}\left|\begin{array}{ccc}
\gamma_{l+2} & \gamma_{l+3} \\
\gamma_{l+3} & \gamma_{l+4}
\end{array}\right|}{\left|\begin{array}{ccc}
0 & \gamma_{l+1} & \gamma_{l+2} \\
\gamma_{l+1} & \gamma_{l+2} & \gamma_{l+3} \\
\gamma_{l+2} & \gamma_{l+3} & \gamma_{l+4}
\end{array}\right|}=\frac{\gamma_{l} \gamma_{l+2}}{\gamma_{l+1}^{2}}\left[1-\frac{\left|\begin{array}{cc}
\gamma_{l+2} & \gamma_{l+3} \\
\gamma_{l+3} & \gamma_{l+4}
\end{array}\right|}{\left|\begin{array}{cc}
\gamma_{l+2} & \gamma_{l+3} \\
\gamma_{l+3} & \gamma_{l+4}
\end{array}\right|+\frac{1}{\gamma_{l+1}^{2}}\left|\begin{array}{cc}
\gamma_{l+1} & \gamma_{l+2} \\
\gamma_{l+2} & \gamma_{l+3}
\end{array}\right|}\right] \geqslant 0 .
$$

This yields

$$
\left[M_{\gamma^{\prime}}\right]_{l}^{2} \text { is positive semidefinite if and only if } 0 \leqslant t \leqslant \frac{-\gamma_{l}\left|\begin{array}{cc}
\gamma_{l+2} & \gamma_{l+3} \\
\gamma_{l+3} & \gamma_{l+4}
\end{array}\right|}{\left|\begin{array}{ccc}
0 & \gamma_{l+1} & \gamma_{l+2} \\
\gamma_{l+1} & \gamma_{l+2} & \gamma_{l+3} \\
\gamma_{l+2} & \gamma_{l+3} & \gamma_{l+4}
\end{array}\right|} \text {. }
$$

Finally from (6.3), (6.4), 6.5) and (6.6) we conclude that

$$
I^{2}=\left[\max \left\{\alpha(P), \alpha(Q), \frac{\gamma_{l}^{2}}{\gamma_{l+1} \gamma_{l-1}}\right\} ; \min \left\{\beta(P), \beta(Q), \frac{-\gamma_{l}\left|\begin{array}{cc}
\gamma_{l+2} & \gamma_{l+3} \\
\gamma_{l+3} & \gamma_{l+4}
\end{array}\right|}{\left|\begin{array}{ccc}
0 & \gamma_{l+1} & \gamma_{l+2} \\
\gamma_{l+1} & \gamma_{l+2} & \gamma_{l+3} \\
\gamma_{l+2} & \gamma_{l+3} & \gamma_{l+4}
\end{array}\right|}\right\}\right]
$$

Remark 2. - The existence of positive roots for $P$ and $Q$ can be checked also by using the classical Bolzano's theorem because of the fact that $P(1) \geqslant 0, Q(1) \geqslant 0$, and $P(0) \leqslant 0, Q(0) \leqslant 0$, and that

$$
\lim _{t \rightarrow \infty} P(t)=\lim _{t \rightarrow \infty} Q(t)=-\infty .
$$

- A direct calculation of discriminants gives the inequality

$$
\min \left\{\gamma_{l-1}^{2} \gamma_{l+1} \gamma_{l+3} ; \gamma_{l-2} \gamma_{l} \gamma_{l+2}^{2}\right\} \geqslant\left(2 \gamma_{l} \gamma_{l+1}-\gamma_{l-1} \gamma_{l+2}\right)^{2} .
$$


6.2. One rank perturbation of weighted shifts. Let $l \in \mathbb{N}$ be given, $t \geqslant$ and let $W_{\alpha}$ be a weighted shift. A $(l, t)$-one perturbation of $W_{\alpha}$ is the weighted shift $W_{\alpha(l, t)}$ defined by $W_{\alpha(l, t)}\left(e_{k}\right)=W_{\alpha}\left(e_{k}\right)=\alpha_{k} e_{k+1}$ for $k \neq l$ and $W_{\alpha(l, t)}\left(e_{l}\right)=\sqrt{t} \alpha_{l} e_{l+1}$.

We denote by $\gamma$ the moment sequence associated with $\alpha$ defined by $\gamma_{0}=1$ and $\gamma_{n}=\alpha_{n-1}^{2} \gamma_{n-1}$ and by $\gamma^{\prime}(t)$ (or simply $\gamma^{\prime}$ ) the moment sequence associated with $\alpha(l, t)$. It is easily seen that $\gamma_{k}^{\prime}=\gamma_{k}$ for $k \leqslant l$ and $\gamma_{k}^{\prime}=t \gamma_{k}$ for $k>l$.

We let

$$
J^{k}=\left\{t \geqslant 0 ; W_{\alpha(l, t)} \text { is } k \text {-hyponrmal }\right\} .
$$

Since $W_{\alpha}$ is $k$-hyponormal if and only if $M_{\gamma}$ is $k$-positive, we conclude that

$$
J^{k}=\left\{t \geqslant 0 ; W_{\alpha(l, t)} \text { is } k \text {-hyponrmal }\right\}=\left\{t \geqslant 0 ; M_{\gamma^{\prime}} \text { is } k \text {-positive }\right\}=I^{k} .
$$

and then from Proposition 3 we see that $I^{k}$ is a compact interval.

The behavior of one rank perturbation of subnormal weighted shift is described by the next theorem.

Theorem 6.1 ([9, Theorem 2.1]). Let $W_{\alpha}$ be a subnormal weighted shift. We have

$$
I^{\infty}:=\cap_{k \geqslant 1} I^{k}=\{1\} .
$$

We state our main result in this section as follows.

Theorem 6.2. Let $W_{\alpha}$ be a k-hyponormal weighted shift. We have

$$
1 \in \stackrel{\circ}{I^{k}} \quad \Longleftrightarrow \quad\left[M_{\gamma}\right]_{k}^{n} \text { is positive definite } \forall n \leqslant l .
$$

We shall use the same notations as in the proof of Proposition 2. We begin with proving that our condition is sufficient. To this aim, we consider

$$
\begin{aligned}
\gamma_{n}^{k}: \mathbb{R}_{+} & \longrightarrow D \\
t & \longmapsto t\left[M_{\gamma}\right]_{k}^{n}+(1-t) H_{k}^{n}(l)
\end{aligned}
$$

Since $\left[M_{\gamma}\right]_{k}^{n} \in \stackrel{\circ}{P_{k}^{+}}$( the set of all positive definite matrices) and $\gamma_{n}^{k}$ is continuous, for $V$ an open neighborhood of $\left[M_{\gamma}\right]_{k}^{n}$ such that $V \subset \stackrel{\circ}{P_{k}^{+}}$, we get the $\left(\gamma_{n}^{k}\right)^{-1}(V \cap D)$ is an open neighborhood of $\left(\gamma_{n}^{k}\right)^{-1}\left(\left[M_{\gamma}\right]_{k}^{n}\right)=1$. Hence, there exists $] r_{n}, t_{n}\left[\subset \mathbb{R}^{+}\right.$such that $\left.1 \in\right] r_{n}, t_{n}\left[\right.$ and $\gamma_{n}^{k}(] r_{n}, t_{n}[) \subset P_{k}^{+}$and then $] r_{n}, t_{n}\left[\subset I_{n}^{k}\right.$. Finally,

$$
1 \in] \max _{n \leqslant l} r_{n}, \inf _{n \leqslant l} t_{n}\left[\subset \stackrel{\circ}{\widehat{I}^{k}} .\right.
$$

Conversely, we assume that there is $n \leqslant l$ such that $\left[M_{\gamma}\right]_{k}^{n}$ is not positive definite, or equivalently, there is $p \leqslant k$ such that $\operatorname{det}\left(\left[M_{\gamma}\right]_{p}^{n}\right)=0$. We distinguish two cases:

$p<k$ : By Theorem $5.4 W_{\alpha}$ is subnormal, then $I^{k}=I^{\infty}=\{1\}$ that means $\stackrel{\circ}{I^{k}}=\emptyset$.

$p=k$ : Simple computations give

$$
\operatorname{det}\left(\gamma_{k}^{n}(t)\right)=t^{k+1} \operatorname{det}\left(\left[M_{\gamma}\right]_{k}^{n}\right)+(1-t) t^{k} \gamma_{n} \operatorname{Cof}\left(\gamma_{n}\right)=\gamma_{n} \operatorname{Cof}\left(\gamma_{n}\right)(1-t) t^{k},
$$

where $\operatorname{Cof}\left(\gamma_{n}\right)$ stands for the $(1,1)$ cofactor of $\left[M_{\gamma}\right]_{k}^{n}$. It follows from $t \in I^{k}$ that $\operatorname{det}\left(\gamma_{k}^{n}(t)\right) \geqslant 0$ and hence $t \leqslant 1$. Thus $1 \notin \stackrel{\circ}{I^{k}}$.

\section{BIBLIOGRAPHY}

1. P.R. Halmos. Normal dilations and extensions of operators // Sum. Bras. Math. 2:9, 124-134 (1950).

2. J. Bram. Subnormal operators // Duke Math. J. 22:1, 75-94 (1955).

3. J. B. Conway. The theory of subnormal operators. Math. Surveys Monogr. 36. Amer. Math. Soc., Providence, RI (1991). 
4. A. Athavale. On joint hyponormality of operators // Proc. Amer. Math. Soc. 103:2, 417-423 (1988).

5. A. Lambert. Subnormality and weighted shifts // J. Lond. Math. Soc. (2) 14:3, 476-480 (1976).

6. J. Stampfli. Hyponormal operators // Pacific J. Math. 12:4, 1453-1458 (1962).

7. R. Ben Taher, M. Rachidi and E.H. Zerouali. Recursive subnormal completion and the truncated moment problem // Bull. Lond. Math. Soc. 33:4, 425-432 (2001).

8. R.E. Curto. Quadratically hyponormal weighted shifts // Integ. Equat. Oper. Theor. 13:1, 49-66 (1990).

9. R.E. Curto and W. Lee. k-hyponormality of finite rank perturbations of unilateral weighted shifts // Trans. Amer. Math. Soc. 357:12, 4719-4737 (2005).

10. N.I. Akhiezer. The classical moment problem and some related questions in analysis, Oliver \& Boyd, Edingburg (1965).

11. R.E. Curto and L.A. Fialkow. Recursively generated weighted shifts and the subnormal completion problem II // Integ. Equat. Oper. Theor. 18:4, 369-426 (1994).

12. D.M. Bressoud. Proofs and Confirmations: The Story of the Alternating-Sign Matrix Conjecture. Cambridge Univ. Press, Cambridge (1999).

13. C. Berg and D. Szwarc. A determinant characterization of moment sequences with finitely many mass-points // Linear Multilinear Alg. 63:8, 1568-1576 (2015).

14. C.E. Chidume, M. Rachidi and E.H. Zerouali. Solving the general truncated moment problem by the r-generalized Fibonacci sequences method// J. Math. Anal. Appl. 256:2, 625-635 (2001).

15. R.E. Curto and L.A. Fialkow. Recursively generated weighted shifts and the subnormal completion problem // Integ. Equat. Oper. Theor. 17:2, 202-246 (1993).

Hamza El Azhar,

Center of mathematical research of Rabat,

Department of Mathematics,

Faculty of sciences,

Mohammed V University in Rabat,

4 Avenue Ibn Batouta,

B.P. 1014 Rabat, Morocco.

E-mail: elazharhamza@gmail.com

Kaissar Idrissi,

Center of mathematical research of Rabat,

Department of Mathematics,

Faculty of sciences,

Mohammed V University in Rabat,

4 Avenue Ibn Batouta,

B.P. 1014 Rabat, Morocco.

E-mail: kaissar.idrissi@gmail.com

El Hassan Zerouali,

Center of mathematical research of Rabat,

Department of Mathematics,

Faculty of sciences,

Mohammed V University in Rabat,

4 Avenue Ibn Batouta,

B.P. 1014 Rabat, Morocco.

E-mail: zerouali@fsr.ac.ma 